der Fallwert war für alle Patienten gleich. Die dadurch entstandene Verwerfung wurde schnell offenbar und kann seit 2010 durch qualifikationsgebundene $\mathrm{Zu}$ satzvolumina (QZV) teilweise korrigiert werden: Tatsächlich gibt es in sehr vielen Kassenärztlichen Vereinigungen (KV) inzwischen allergologische QZV zumindest für manche Facharztgruppen.

Seit diesem Jahr gibt es darüber hinaus erstmals die Möglichkeit, so genannte „förderungswürdige Leistungen“ $\mathrm{zu}$ definieren. Diese werden außerhalb von RLV und QZV gesondert vergütet. Immerhin sechs KV-Bezirke haben bisher die allergologische Diagnostik oder die subkutane spezifische Immuntherapie als förderungswürdig anerkannt. $\mathrm{Zu}-$ letzt sind zum 1. Oktober 2013 die Bundesländer Sachsen und Brandenburg dazugekommen.

Auf dieser Doppelseite finden Sie eine bundesweite Übersicht über die KV-Bezirke mit allergologischen QZV (Tab. 1) sowie über die KV-Bezirke, in denen allergogolgische EBM-Ziffern bereits als besonders förderungswürdig definiert wurden (Tab. 2). Die gezeigten Daten sind öffentlich zugänglich und geben den Stand zum 23. Oktober 2013 für das Quartal 4/2013 wieder.

\section{KVen, die allergologische Gebührenordnungspositionen in den Katalog förderungswürdiger Leistungen aufgenommen haben}

Tabelle 2

KV

Baden-

Württem-

berg

Branden- $\quad 01.10 .201330130$ Die Höhe der Zuschläge/Zusatzpunktwerte ermittelt sich auf Basis

burg

\section{gültig seit GOP Vergütungsregelung}

01.01.2013 30130 Förderungsbetrag 3,00€/Leistung, max. 12-mal im Behandlungsfall

Nordrhein

01.01.2013

30110

\section{1}

30120

30121

30122

30123

\begin{tabular}{l|l|l|l|}
\hline Sachsen & 01.10 .2013 & 30130 \\
\hline
\end{tabular}

30131

Schleswig- 01.04.2013 30130

Holstein

Thüringen 01.01 .2013
30131

30120

30121

30122

30123

$3,29 €$ Zuschlag $^{3}$ den Volumens in Höhe von gruppenspezifische Leistungen eine Staffelung der Höhe der Zusatzpunktwerte in verschiedenen Klassen, die gewährleistet dass der Anteil der Arztgruppe den entsprechenden Anteil am Leistungsbedarf des Honorarfonds Fachärzte nicht übersteigt.

Punktwertzuschlag HNO und Derma 2,64 Ct, Pneumo 1,25 $\mathrm{Ct}^{1}$ Punktwertzuschlag HNO und Derma 2,64 Ct, Pneumo 1,25 Ct ${ }^{1}$ Punktwertzuschlag HNO und Derma 2,64 Ct, Pneumo 1,25 Ct ${ }^{1}$ Punktwertzuschlag HNO und Derma 2,64 Ct, Pneumo $1,25 \mathrm{Ct}^{1}$ Punktwertzuschlag HNO und Derma 2,64 Ct, Pneumo 1,25 $\mathrm{Ct}^{1}$ Punktwertzuschlag HNO und Derma 2,64 Ct, Pneumo 1,25 Ct ${ }^{1}$ Punktwerterhöhung von $1,85 \mathrm{Ct}$ für $4 / 2013$ und $1 / 2014^{2}$ Punktwerterhöhung von $1,85 \mathrm{Ct}$ für $4 / 2013$ und $1 / 2014^{2}$ $1,87 €$ Zuschlag $^{3}$

$1,41 €$ Zuschlag $^{3}$

$1,31 €$ Zuschlag $^{3}$

$3,29 €$ Zuschlag $^{3}$

$9,37 € /$ Injektion, keine Mengenbegrenzung

$301317,07 €$ pro GOP, keine Mengenbegrenzung
'Werte für Quartal 1/2013, Punktwertzuschläge für Quartale 2-4/2013 floatend;

ºhne Bereinigung der QZV, ab 2/2014 Neubewertung;

${ }^{3}$ Der Leistung muss seit 2009 eine Provokation vorangegangen sein

Stand: 23. Oktober 2013

\title{
Allergiker in Deutschland sind unterversorgt - erste Lösungsansätze
}

Im Rahmen des 8. Deutschen Allergiekongresses in Bochum im September präsentierte das "Aktionsforum Allergologie" erstmals die Ergebnisse der sogenannten "Wasem-Studie“ der Öffentlichkeit. Dabei handelt es sich um die bisher größte deutsche Versorgungsstudie aus dem Bereich der Allergologie. Der Gesundheitsökonom Prof. Dr. Jürgen Wasem von der Uni Duisburg-Essen hatte die Daten von 40 Millionen Versicherten im Zeitraum von 2007 bis 2010 analysiert und war dabei zu ernüchternden Ergebnissen gelangt: Obwohl in Deutschland rund 20 Millionen Menschen unter Allergien leiden, wird ein Großteil davon nicht ausreichend oder gar nicht versorgt.

Prof. Dr. Wolfgang Wehrmann hatte bereits 2012 gemeinsam mit weiteren Fachspezialisten mit einem viel beachteten Aufsatz in der Zeitschrift Allergologie auf den Sachverhalt und die möglichen Ursachen hingewiesen [Allergologie 2012; 35: 473-9]. Dazu gehören u.a. der demographische Wandel, Defizite in der ärztlichen Aus- und Weiterbildung, Angst vor Arzneimittelregressen und eine unzureichende Würdigung der Allergologie in den ärztlichen Honorarsystemen.

Eine Lösung dieser Problematik kann nur gemeinsam erarbeitet werden. Vor diesem Hintergrund ist die Gründung des bundesweiten „Aktionsforums Allergologie" zu sehen. In diesem Forum haben sich über die beiden großen allergologischen Verbände AeDA und DGAKI hinaus auch die Berufsverbände der HNO-Ärzte, der Pneumologen, der Dermatologen und der Kinder- und Jugendärzte zusammengefunden, um Versorgungsprobleme in der Allergologie gemeinsam anzugehen und zu lösen. Aktuelle Informationen dazu bietet die Website des Forums unter www.aktionsforum-allergologie.de.

In Sachsen ist es der Kassenärztlichen Vereinigung in Kooperation mit den Landesvorsitzenden der Berufsverbände der HNOÄrzte, der Dermatologen und der Pneumologen gelungen, eine angemessene Regelung für die Vergütung allergologischer Leistungen in der Honorarverteilung zu finden: Diese werden seit dem 3. Quartal 2010 im Rahmen von QZV und seit dem 1. Oktober 2013 auch als förderungswürdige Leistung vergütet. In vielen Gesprächen haben die Landesvorsitzenden die Versorgungsprobleme von Allergikern gegenüber der KV Sachsen und den Krankenkassen transparent dargestellt und die Notwendigkeit von Maßnahmen zur Förderung der Allergologie vermittelt. Das gemeinsame Engagement wird auch im Hinblick auf die regionalen Honorarverhandlungen 2014 fortgesetzt. Aus der Vollpublikation der Wasem-Studie [Allergo J 2013, 22: 366-73] ergeben sich dazu ausreichende Argumente.

\section{Dr. Sylvia Krug}

Deutscher Berufsverband der HNO-Ärzte, Landesgruppe Sachsen 